\title{
QUANTITATIVE ANALYSIS OF IMMUNOFLUORESCENT RETINAL IMAGES
}

\author{
Jiyun Byun, Nhat Vu, Baris Sumengen and B. S. Manjunath \\ Center for Bio-image Informatics \\ Department of Electrical and Computer Engineering \\ University of California, Santa Barbara, CA 93106-9560 \\ http://www.bioimage.ucsb.edu/
}

\begin{abstract}
We present a novel method to quantitatively analyze confocal microscope images of retinas. We automatically detect nuclei within the outer nuclear layer (ONL) in a retinal image. Based on nuclei detection results, we also automatically measure the thickness of the ONL and the local cell density within the ONL. These measurements provide the first thorough quantitative analysis of retinal images. Our results not only verify previous conclusions about retinal restructuring during detachment, but also provide biologists with significant information about the regional responses in the ONL.
\end{abstract}

\section{INTRODUCTION}

In recent years, much progress in bio-molecular imaging technologies have allowed biologists to generate vast amounts of microscopic images of cells and tissues quickly. Analysis of these images is critical for understanding behaviors of complex biological systems. However, much of the image interpretation is currently done manually, which is time consuming and not easily reproducible or converted to quantitative measures useful to other biologists. Therefore, it is necessary to utilize image analysis methods that are fast and that can provide reliable quantitative results.

In this paper, we focus on analyzing confocal micro scope images of retinas taken during detachment experiments. To provide biological background, the retina is the light-sensitive layer of tissue that lines the inside of the eye and is composed of three layers of nerve cell bodies and two layers of synapses. The main function of this complex tissue structure is to send visual messages through the optic nerve to the brain. When the retina detaches, it is lifted or pulled from its normal position. If not promptly treated, retinal detachment can cause permanent vision loss. Understanding the mechanisms behind the loss and recovery of vision following retinal detachment has been the focus of studies for many years [1]. Photoreceptors have received the greatest attention

This research is supported by NSF \# ITR-0331697, Nhat Vu is supported by NSF IGERT \# DGE-0221713, and the data collection is supported by NIH \# EY-00888.
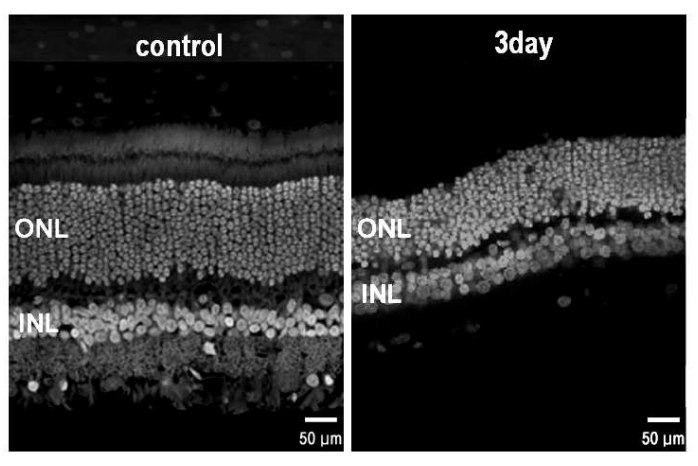

Fig. 1. Confocal images of normal (left) and detached (right) retinas stained with TOPRO.

in these studies since photoreceptor outer segment degeneration is considered the primary effect of detachment (Fig.1), and qualitatively, it was verified that the ONL appears to be much more loosely packed with nuclei as a result of cell loss following detachment [1]. Degeneration of the photoreceptors has been measured in various ways $[1,2,3]$ : the number of rows of nuclei in the ONL, the area of the ONL, the thickness of the ONL, and the number of nuclei. The change in these measurements over time is used as an index of photoreceptor degeneration.

In past works, algorithms developed for automatic micrograph analysis have been very task specific and are not easily extensible to analysis of immunofluorescent retinal images. For example, Malpica [4] used watershed to segment clustered nuclei, Nedzved [5] used morphological operators to segment cells in images with sparse density, and Boland [6] used a neural network to classify subcellular structures in HeLa cells. However, these tools were not designed to analyze immunofluorescent retinal images, which present unique challenges-cells are densely packed (600 cells in 512 x 100 pixels), uneven staining causes intensity variations within cell layers, and non-uniform DNA distribution inside the nucleus causes intensity variations within individual cells. Therefore, there is a need to develop new methods to analyze this type of images.

The rest of the paper is organized as follows. In section 2 , 
we discuss the detection of fluorescent photoreceptor nuclei from confocal images of control and 3-day detached retinas. Section 3 describes the method developed to measure both ONL thickness and density profiles based on the detected nuclei. In section 4 , we present and compare our results with those obtained manually, and we conclude the paper with a brief discussion in section 5 .

\section{NUCLEI DETECTION}

\subsection{Nuclei detector design}

Our study focuses on developing an efficient detection solution for a class of blob-like structures-roughly convex local intensity distributions whose iso-level contour are approximately ellipsoidal with some irregularities that do not destroy the ellipsoidal topology. The intensity distribution of some nuclei are multi-modal because of inconsistent staining and biological characteristics of DNA distribution within a nucleus. However, the intensity distribution can be assumed to be uni-modal under appropriate amounts of Gaussian blurring. Therefore, a blob can be modeled as a 3-D surface generated by rotating a ridge profile around its central axis. If we model the nuclei as a blob with some additive Gaussian noise, we can design an optimum linear blob detector by rotating the second derivative of a Gaussian around its central axis.

In our work, we utilize the inverted Laplacian of Gaussian (LoG) as our blob detector. We modeled the nuclei as circular objects although these objects mostly resemble ellipsoids. Our objective in using a circular model, a very general shape model, is to achieve a rotation invariance in our detection. Otherwise it is possible that we are overfitting our design to the training images. Once we have a blob detector, the blob centers are detected with the following two steps:

Blob detector design: The LoG filter is designed such that the diameter of the filter is proportional to the average diameter of nuclei in the image. For example, cell bodies in the feline retina range from 4-6 $\mu \mathrm{m}$ in diameter, so the size of the filter is set to $5 \mu \mathrm{m}$. The given retinal image is filtered by the inverted LoG producing a smooth continuous image in which the local maxima correspond to blob centers.

Search for local maxima: We search the filtered output for local maxima. The minimum distance between blob centers is used as the search radius for the local maxima, and this parameter is defined to be proportional to the filter size.

\subsection{Evaluation of the nuclei detector}

Since we use the detected nuclei information to measure the thickness and local cell density of the ONL, it is important to obtain accurate and reliable detection results. The goal of the nuclei detector is to count the number of nuclei within the ONL accurately close to that of manual counting. The manual counting results, which we call ground truth, are compared to the results of the automatic nuclei detector using various combinations of parameters. To estimate optimal parameters of the nuclei detector, we evaluate its performance using an error criterion defined as

$$
E \triangleq \frac{1}{N} \times \frac{|N D-G T|}{G T}
$$

where $\mathrm{N}$ is the number of images in the training set,ND and GT are the numbers of nuclei detected by our detector and by manual counting, respectively.

Because it takes a considerable amount of time to count the number of nuclei manually, we obtained ground truths for only 41 retinal images and, due to our small dataset, we employ the leave-one-out cross-validation method to evaluate the detector's performance. That is for each of 41 runs, we leave out one image from the dataset and optimize the detector based on its performance on the remaining images. Then we apply the resulting detector to the entire image set and compute its error. From the 41 detectors optimized for each run, the detector with the best performance is chosen.

The proposed nuclei detector uses a Laplacian of Gaussian filter and searches the local maxima of the filter output. Therefore, the performance depends on two factors: 1) how well the filter approximates the shape of a nucleus which is determined by the filter size and the standard deviation $\sigma$, and 2) how well the local maxima are determined, which is dependent on the minimum distance between blob centers. Since we search the filter output for local maxima, $\sigma$ does not affect the performance of the nuclei detector. Thus, we vary two parameters: cell size $(c s)$ in $\mu m$ and the proportion $(p)$ of the minimum distance between blob centers and filter size $(f s)$, where $f s$ is defined as $c s \times$ the pixel resolution of a given image. The parameter $c s$ is varied by five different values from 4 to 8 . The range of the values is determined by the cell size within the ONL. The parameter $p$ varies by ten different values from 0.1 to 1 . Thus, we have a total of $5 \times 10$ combinations, and 41 runs are conducted for each combination. We find the nuclei detector achieves a maximum performance with $c s=5 \mu m$ and $p=0.5$. In practice additional training is not needed, and this detector can be used for any type of feline retinal images given that the image resolution is known.

\section{LOCAL THICKNESS AND DENSITY}

\subsection{Median extraction}

To calculate the local thickness and density, the precise ONL boundary must be extracted from the image. We convert the $(\mathrm{x}, \mathrm{y})$ coordinates of detected nuclei centers into on pixels in a binary image. The ONL extraction is performed by two morphological operations on the binary image. First, a closing operation on the nuclei centers provides an approximation of the ONL. We use a disk with diameter approximately six times the size of the photoreceptor as the structuring element. 
The relatively large diameter size ensures that the ONL can be extracted without any holes. Second, the morphological closing result is dilated by half a photoreceptor size, thereby extending the ONL to encompass the nuclei on the layer boundaries. Because there are large variations in intensity within the ONL, segmenting the ONL using the nuclei detection results ensures that every detected photoreceptor nucleus is properly included within the ONL.

The ONL boundary is not naturally smooth and is usually curved along its length, therefore we compute a median curve and use it as the basis for the thickness and density measurement. Having segmented the ONL, we compute the median curve by pruning the skeleton of the ONL. The median extraction is summarized below. First, we use the divergenceordered thinning algorithm proposed by [7] to calculate the total outward flux for each ONL. For every pixel $p$ inside the ONL, the Euclidian distance $d(p)$ from $p$ to the nearest boundary pixel is calculated. The result is a distance map $D$ shown in Fig. 2 (a). Next we compute the unit gradient of $D$ using the $\vec{g}(p)=\nabla D(p) /|\nabla D(p)|$. From $\vec{g}(p)$, we calculate the total outward flux given by $\Phi(p)=\sum_{i=1}^{8}<\vec{g}\left(p_{i}\right), \vec{n}_{i}>$, where $p_{i}$ is an 8-connected neighbor of $p$ and $\vec{n}_{i}$ is the unit vector pointing from $p$ to $p_{i}$. The total outward flux result is shown in Fig.2 (b). At pixel locations near the median, the gradient $\vec{g}(p)$ diverges and thus the flux $\Phi(p)$ is positive. Conversely, at locations where the gradient converges, the flux is negative.

Using the outward flux map, the rough skeleton of the ONL is extracted by truncating all negative values of $\Phi(p)$ and thresholding the truncated result. The threshold level is chosen to minimize the variance of values above and below the threshold. Fig.2 (c) shows the result of this skeletonization step. We prune the skeleton by removing side branches to extract the median curve. Information from the outward flux result is used to determine which side branch to disconnect. The final median curve is smoothed using cubic-spline interpolation. The smoothing is necessary to filter out noisy pixels resulting from the skeletonization.

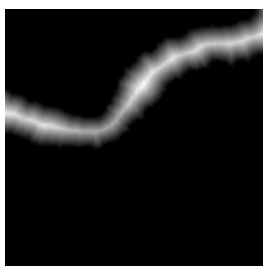

(a)

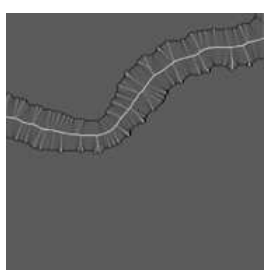

(b)

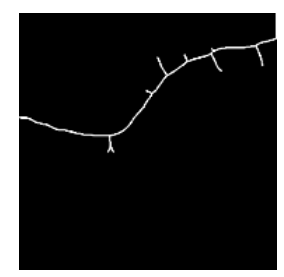

(c)
Fig. 2. Median extraction.(a) Distance map $D$. (b) Total outward flux $\Phi(p)$. (c) Skeleton after thresholding.

\subsection{Thickness and density profile}

Once the median curve is extracted, it can be used to measure the thickness and density along the entire length of the ONL. For every location $s_{i}$ along the median curve, a line $l\left(s_{i}\right)$ orthogonal to that median location is constructed and extended outward to the ONL boundaries (Fig.3 (c)). The thickness at location $s_{i}$ is defined as the length of $l\left(s_{i}\right)$. Thickness measurements along the entire length of the median provide a continuous thickness profile as shown in Fig.3 (d).

To compute the density at each median location $s_{i}$, a sliding region $A\left(s_{i}\right)$ bounded by $l\left(s_{i}-k d s\right)$ and $l\left(s_{i}+k d s\right)$ is constructed, where $k$ is an integer parameter controlling the size of $A\left(s_{i}\right)$ and $d s$ is the finite length element between adjacent median points (Fig.3 (c)). The density is defined as the number of nuclei located inside $A\left(s_{i}\right)$ divided by the area of $A\left(s_{i}\right)$. Fig.3 (d) shows the thickness and density profiles of the ONL in Fig.3 (a). The vertical line in the profile plots indicates the thickness and density of the ONL at location $s_{i}$ in Fig.3 (c).

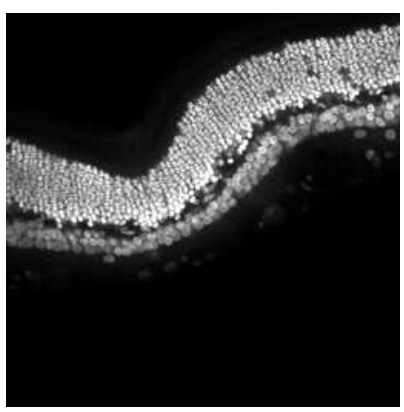

(a)

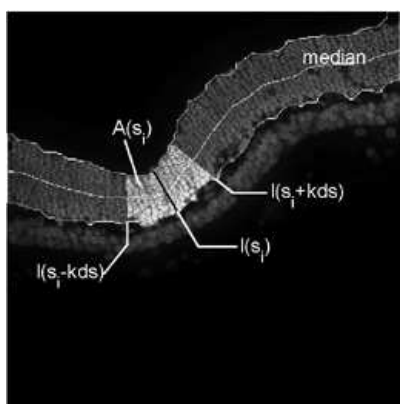

(c)

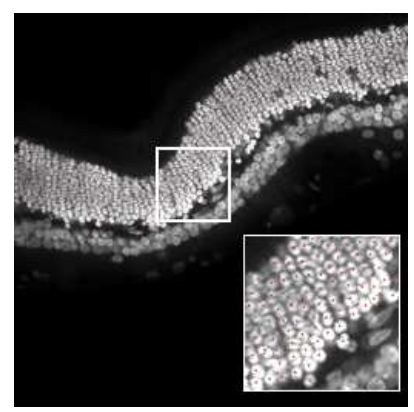

(b)

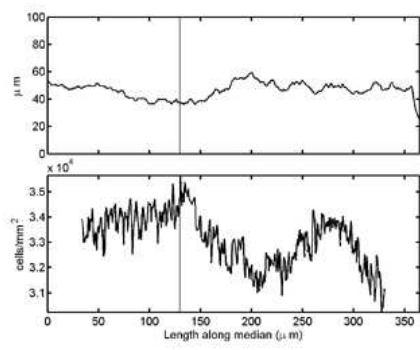

(d)
Fig. 3. Analysis result. (a) Confocal image of a 3-day detached cat retina. (b) Cell detection result. Detected cell centers are marked. (c) The ONL with median curve, $\vec{l}\left(s_{i}\right)$, and highlighted region $A\left(s_{i}\right)$. (d) Thickness profile (top) and density profile (bottom).

\section{EXPERIMENTAL RESULTS}

We present the results of applying the proposed method to a series of confocal images. 21 control and 20 3-day detached feline retinal images were collected from tissue sections stained with TOPRO using an Olympus FluoView laser scanning confocal microscope. To detect nuclei within the ONL, we first create a mask of the rough boundary of the ONL for each image. This mask is automatically created by median filtering followed by morphological operations. Then we apply the optimized nuclei detector to analyze the data 
set. Manual counting results from the same data set are used as ground truths. To obtain the ground truths, each image was manually counted three times independently to measure intraobserver variation. The average variation over 41 images was $1.8 \%$ ranging from 0.2 to $5.35 \%$.

To evaluate the performance of the nuclei detector, the automatic detection results are compared with the ground truths. The automated nuclei detector approximates the number determined by manual counting with an average error of $3.67 \%$. Fig.3 (b) shows the locations of detected nuclei centers overlayed on the image. After nuclei detection, we generate the ONL thickness and density profiles for each image. For each ONL thickness profile, we calculate the mean and standard deviation. To measure the global change in ONL thickness, we average the mean thicknesses over all images under the same experimental condition.

The quantitative cell counts and ONL thickness measurements confirm that the number of photoreceptors in the ONL decreases in response to retinal detachment. This is the first time that a thorough quantitative analysis has been done, verifying earlier qualitative predictions in [1]. Fig.4 (a) shows the average number of photoreceptor nuclei per $\mathrm{mm}^{2}$ in 21 control and 203 -day detached retinal images obtained by manual counting and automatic nuclei detection. The control retinas have, on average, 37503 nuclei $/ \mathrm{mm}^{2}$. After 3 days of detachment, this number is significantly reduced to an average of 33590 nuclei $/ \mathrm{mm}^{2}$ ( $\left.\mathrm{p}=0.0001\right)$. Even though the average number of photoreceptor nuclei differs slightly from the manual count, the nuclei detector captures relative differences in cell density between the control and 3-day detached cases and reaches the same conclusion as that derived from manual counting. Similarly, results in Fig.4 (b) shows that the average ONL thickness significantly decreases from $66.9874 \mu \mathrm{m}$ to $49.4947 \mu m$ after detachment $(\mathrm{p}=0.01)$.

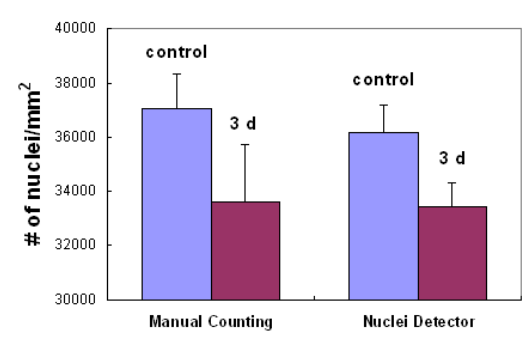

(a)

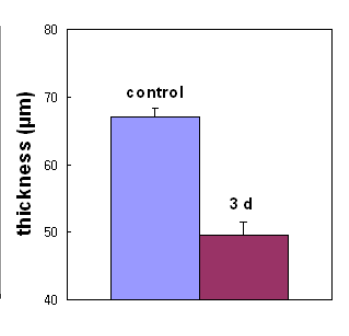

(b)
Fig. 4. (a) A comparison of manual and nuclei detector counts for 41 images. (b) Thickness of the ONL.

\section{DISCUSSION AND CONCLUSION}

In this paper, we present novel methods to analyze immunofluorescent retinal images: 1) we designed a nuclei detector capable of identifying photoreceptor centers and evaluated its performance, and 2) using the nuclei detection result, thickness and density profiles were computed for each ONL. Re- sults obtained from our methods, the first thorough quantitative analysis for retinal images, not only corroborated conclusions derived from manual measurements and qualitative assessments, but our analysis also provides significant information about local structural changes during retinal detachment. Specifically, we will use our results to find correlations between thickness and density at different locations in the ONL and quantitatively measure how these regions in the retina respond differently to detachment. These analysis will help biologists to target specific areas of the retina for treatments, such as retinal reattachment.

\section{ACKNOWLEDGEMENTS}

We would like to thank Dr. Geoffrey Lewis, Dr. Mark Verado, and Prof. Steven Fisher from the Neuroscience Research Institute for the retinal image dataset and for their helpful discussions, Camille Cazon for manual counting, and Kallen Betts for the sample preparation.

\section{REFERENCES}

[1] S. K. Fisher, G. P. Lewis, K. A. Linberg, and M. R. Verardo, "Cellular remodeling in mammalian retina: results from studies of experimental retinal detachment," Progress in Retinal and Eye Research, vol. 24, pp. 395431, 2005.

[2] Z.Y. Li, M. O. Tso, H. M. Wang, and D. T. Organisciak, "Amelioration of photic injury in rat retina by ascorbic acid.," Investigative Ophthalmology and Visual Sciences, vol. 26, no. 1589, 1985.

[3] J. J. Michon, Z. Li, N. Shioura, R. J. Anderson, and M. O. M. Tso, "A Comparative study of Methods of Photoreceptor Morphometry," Investigative Ophthalmology and Visual Sciences, vol. 32, no. 2, pp. 280-284, 1991.

[4] N. Malpica, C. O. de Solorzano, J. J Vaquero, A. Santos, I. Vallcorba, J. M. Garcia-Sagredo, and F. del Pozo, “Applying watershed algorithms to the segmentation of clustered nuclei.," Cytometry., vol. 28, no. 4, pp. 289-297, 1997.

[5] A. Nedzved, S. Ablameyko, and I. Pitas, "Morphological segmentation of histology cell images," ICPR, vol. 1, pp. 500-503, 2000.

[6] M. Boland and R. Murphy, "A neural network classifier capable of recognizing the patterns of all major subcellular structures in fluorescence microscope images of HeLa cells," Bioinformatics, vol. 17, no. 12, pp. 1213-1223, 2001.

[7] P. Dimitrov, C. Phillips, and K. Siddiqi, "Robust and efficient skeletal graphs," in Conference on Computer Vision and Pattern Recognition, 2000. 